\title{
Restrictions on Extended Gravity at Galaxy Clusters Scales
}

\author{
Stanislav Alexeyev ${ }^{1,2, *}$, Boris Latosh ${ }^{3,4}$, and Vsevolod Echeistov ${ }^{5}$ \\ ${ }^{1}$ Sternberg Astronomical Institute, Lomonosov Moscow State University, Universitetsky Prospekt, 13, \\ Moscow 119234, Russia \\ ${ }^{2}$ Department of Quantum Theory and High Energy Physics, Physics Faculty, Lomonosov Moscow State \\ University, Vorobievi Gori, 2/1, Moscow 119234, Russia \\ ${ }^{3}$ Department of Theoretical Physics, Faculty of Natural and Engineering Science, Dubna International \\ University, Universitetskaya Str., 19, Dubna, Russia \\ ${ }^{4}$ Department of Theoretical Physics, Faculty of Physics and Astronomy, University of Sussex, Brighton, \\ BN1 9QH, United Kingdom \\ ${ }^{5}$ Department of Astrophysics and Stellar Astronomy, Physics Faculty, Lomonosov Moscow State Uni- \\ versity, Vorobievi Gori, 2/1, Moscow 119234, Russia
}

\begin{abstract}
Following [1] we discuss the predictions of Starobinsky model of $f(R)$-gravity with vanishing cosmological constant at galaxy and galaxy clusters scales. As a result we demonstrate that at the current observational accuracy level there is no significant difference in cut-off radius values for Starobinsky model and general relativity (GR) in the mass range from $10^{9} M_{S \text { un }}$ till galaxy clusters ones $\left(10^{18} M_{S u n}\right)$ that shows the good applicability of GR at these ranges.
\end{abstract}

\section{Introduction}

The general relativity (GR) successfully describes all observable gravitational phenomena on the scales of the solar system [2]. The discovery of gravitational waves [3] confirmed the applicability of GR for the description the gravitational interaction [4] over a wide range of distances. Nevertheless, there is reason to believe that GR is not able to provide a fully correct description of gravity on large scales and in the regime of huge energies. The Universe accelerated expansion [5] could serve as an indication of the incomplete astrophysical applicability of GR. This accelerated expansion most accurately is described by adding a cosmological constant. As a consequence, the introduction of an additional constant term to the action causes difficulties in matching with the quantum theory [6, 7]. The limited applicability of GR in the high-energy regime can be proved by several phenomena. Firstly, GR is not renormalizable [8], so, in the quantum regime it could be only an effective theory [9]. Further, self-consistent cosmological models require the presence of an inflationary stage [10]. To resolve these problems the gravity was extended by additional fields and curvature expansions [11-13]. We discuss scalar-tensor extension of GR and its particular case: $f(R)$-gravity [14]. The choice of function $f$ is not arbitrary $[15,16]$. Lagrangians of such type appear also during taking into account the radiative corrections of quantum field theory in curved space-time [17].

*e-mail: alexeyev@sai.msu.ru 
It is interesting that as the Newtonian gravity can be treated as asymptotes of a more general theory of gravity (GR) in weak gravitational fields we can assume that GR itself is an approximation of a more general theory at solar system scales. Such approach allows to introduce the different extended gravity for the description of dark matter and dark energy. Some models treat dark energy as space-time geometry: massive gravity, theories with violation of Lorentz invariance (under extreme conditions), or, theories with additional fields or space-time dimensions $[11,18]$. Keeping in mind the relationship between $f(R)$ gravity and scalar-tensor ones [19], as a first step we consider the model proposed in [16]. At this model the function $f(R)$ must be regular and vanishes for $R=0$. Models where the function $f$ diverges in the small curvature region are indistinguishable from GR or can not give a description of the existing data [16]. In addition, the scale of the curvature $\Lambda$ is interpreted as the magnitude of the effective cosmological constant. When $R \gg \Lambda$, the function $f$ tends to $R-2 \Lambda$. Following [1], we refer the considered as Starobinsky one.

In [16] the function $f$ is suggested as:

$$
f(R)=R+\lambda R_{0}\left(\left(1+\frac{R^{2}}{R_{0}^{2}}\right)^{-n}-1\right),
$$

where $R$ is the scalar curvature, $\lambda, R_{0}$ and $n$ are the model specific parameters. The case $\lambda>0$, and $R_{0}$ coincides with the cosmological constant in order of magnitude. As shown in [16] for large values of curvature (effective) the cosmological constant is expressed as:

$$
\Lambda=\frac{\lambda}{2} R_{0} .
$$

Therefore we fix $\lambda=2$ and $R_{0}=\Lambda$.

According to [16], in the intermediate mode $(0<R<\Lambda)$ the Starobinsky model differs from GR and can describe the dark energy effects. Laboratory tests and observations in the solar system provide the following limitation: $n \geqslant 2$. The model should describe gravitational phenomena on all scales; therefore we study its manifestations on the scales of galaxies and clusters of galaxies. In order to check the model, it is necessary to use values obtained with the help of real observational data. At this level, the Universe expansion manifests itself as a force acting equally on all bodies and preventing their gravitational connection. The balance of the force of gravitational attraction and the force associated with the accelerated expansion defines a specific scale called as "the turning radius". It determines the maximum size of the gravitational bound system [20,21]. Thus, it is interesting to compare the turning radii predicted by GR and Starobinsky model and with the observed spatial scales of galaxies and galaxy clusters.

\section{Turning radius calculation}

The spheroidal and some elliptical galaxies, as well as the so-called regular clusters of galaxies, have a close to spherical shape, small rotational velocities (in comparison with the dispersion of ones), and a central part [22-24]. Therefore, as a first approximation step to describe the gravitational field in the galaxy, one can start from a spherically symmetric geometry, assuming that the most part of the mass is concentrated in the center. So, we use Schwarzschild coordinates represented in the form:

$$
d s^{2}=e^{A} d t^{2}-e^{-A} d r^{2}-r^{2} d \Omega
$$

where $A=A(r)$ is unknown function. 
Field equations in $f(R)$-gravity are $[14,16]$ :

$$
f^{\prime}(R) R_{\mu \nu}-\frac{1}{2} f(R) g_{\mu \nu}-\left(\nabla_{\mu} \nabla_{v}-g_{\mu \nu} \square\right) f^{\prime}(R)=0,
$$

where $f^{\prime}(R)=d f(R) / d R, \nabla_{\mu}$ is covariant divergence, $\square=\nabla_{\mu} \nabla^{\mu}$ is d'Alambert operator. Eqs ((4)) represent nonlinear second order system. We solve it numerically. As a first step we decrease the equations order by a new variable $a$ :

$$
a=\frac{d A}{d r} .
$$

Similarly we assume the scalar curvature as the independent variable. In the role of coupling equation for $A$ and $a$ we use its explicit form:

$$
R=-\frac{2}{r^{2}}+e^{A}\left(\frac{d a}{d r}+a^{2}+\frac{4 a}{r}+\frac{2}{r^{2}}\right) .
$$

From $(1,1)$-component of Eqs ((4)) it is possible to extract $d^{2} f / d r^{2}$ and with $(0,0)$ component of ((4)), to show that:

$$
\frac{d R}{d r}=\frac{f^{\prime}(R) \cdot R_{00} / g_{00}-f(R) / 2}{\left(e^{A}(a+2 / r)+a / 2\right) f^{\prime \prime}(R)} .
$$

So, we work with the equations $((5)),((6))$ and (7) together:

$$
\left\{\begin{array}{l}
\frac{d A}{d r}=a, \\
\frac{d a}{d r}=e^{-A}\left(R+\frac{2}{r^{2}}\right)-a^{2}-\frac{4 a}{r}-\frac{2}{r^{2}}, \\
\frac{d R}{d r}=\frac{f^{\prime}(R) \cdot R_{00} / g_{00}-f(R) / 2}{\left(e^{A}(a+2 / r)+a / 2\right) f^{\prime \prime}(R)},
\end{array}\right.
$$

where $f$ is defined in ((1)) and Ricci tensor components are calculated using the metrics ((3)).

The system ((8)) is solved with the help of the modified Runge-Kutta method (TR-BDF2, [25]). The mass spectrum begins at $10^{9} M_{S u n}$ and lied till $10^{18} M_{S u n}$ as these values that are typical for individual galaxies (Mass of the Milky Way Galaxy -4 times $10^{11} M_{S \text { un }}$ [26]) and for clusters (the characteristic masses of the clusters are $\sim 10^{15} M_{S \text { un }}$ [27-29]) respectively. We checked $n$ range from 1 to 9 using the following definition of the gravitational potential:

$$
\phi=\frac{c^{2}}{2}\left(g_{00}-1\right)=\frac{c^{2}}{2}\left(e^{A}-1\right),
$$

as well as its dimensionless version:

$$
\phi / c^{2}=\frac{e^{A}-1}{2} .
$$

The turning radius is located at the point where the force of gravitational attraction is equal to the one coming from the Universe accelerated expansion. At this point the gravitational potential must vanish:

$$
\frac{d \phi}{d r}=0
$$

Since $a$ is an independent variable, one can search $r_{1}$ and $r_{2}$ for which $a\left(r_{1}\right)>0$ and $a\left(r_{2}\right)<0$, respectively. Next step is to calculate the desired value of the turning radius using the linear 
interpolation. The dependence of the radius against the object mass for Starobinsky's model with various $n$ values and GR is shown in Fig. 1. The dependencies are very close to the power law, so, on a logarithmic scale they are linear. We approximate the dependence of the radius $R_{T A R}$ of the turning one by:

$$
R_{T A R}=M^{\alpha} \times R_{T A R}\left(M_{S o l}\right),
$$

where $R_{T A R}$ is measured in kiloparsecs $(k p c)$ and the mass of the object $M$ is expressed in the Sun ones $\left(M_{S \text { un }}\right)$. In this logarithmic form:

$$
\log _{10}\left(R_{T A R}\right)=\alpha \log _{10}(M)+\beta .
$$

We present the values of the ratio of the rotation radii for the Starobinsky model and GR against the mass in Fig. 2. In the complete range of considered masses, the Starobinsky models with $n=1$ and $n=2$ have the least radii of zero mass, and so they should be compared with observations first of all.

\section{Comparing with observational data}

Direct measurements of the turning radius are impossible yet. To proceed this it requires to observe directly the motion of a test body (a star) in a distant galaxy. Such observations are still unreal. To avoid this problem it is possible to take the density profile obtained from gravitational lensing. One can use it as an infinum value of the object size. The turning radius limits the maximum object size. It cannot be less than the density profile size. Similar profiles were obtained from the analysis of CLASH $[27,28]$ and other clusters of galaxies [29]. All this is comparable with $n=1$ and $n=2$ Starobinsky models and also with GR.

Despite significant differences, none of the discussed models (even with $n=1$ that can not pass solar system tests [16]) deviate more than by $20 \%$ from the radius determined by GR and fixed by the observational data. Thus, the current level of observational accuracy does not allow strengthening or confirming the earlier limitations on the Starobinsky model.

\section{Discussion and Conclusions}

We considered the $f(R)$ - gravity with vanishing cosmological constant (the Starobinsky model) on scales of galaxies and their clusters. Turning radius values for masses of galaxy clusters are obtained, as a strict upper bound on the size of the clusters. It was found that the dependence of the turning radius against the mass of the object for the Starobinsky model differs from that obtained in GR very little. The turning radius increases with increasing of $n$ in all mass ranges. Despite the fact that some models give smaller turning radius than GR, no model exceeded the limits set by observational data: $20 \%$ of the radius given by GR.

Concluding we see that the achieved level of accuracy does not allow obtaining additional restrictions on the Starobinsky model with a vanishing cosmological constant on the scales of clusters of galaxies. Thus, at the existing level of accuracy, the Starobinsky model and, consequently, the scalar-tensor models associated with this one by the transformations do not contradict the astronomical data on the scales of galaxies and their clusters. We also want to emphasize the perspectives of the proposed method in increasing its own accuracy and its usage for testing other extended gravity models.

\section{Acknowledgements}

The work was supported by the RFBR grant 16-02-00682. The authors are grateful to Maxim Pshirkov for interesting discussions and critical remarks. 


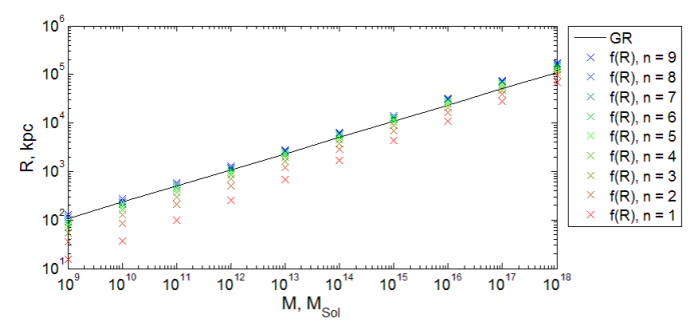

Figure 1. Dependence of the radius against the mass for the Starobinsky models with $n=1 . .9$ (from the bottom to the top), as well as GR (direct line) in the logarithmic scale

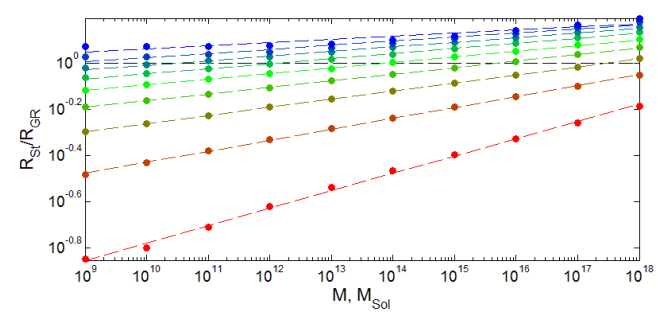

Figure 2. The ratio of the rotation radius of the Starobinsky model to the same value in GR against the mass. Here the points represent the results of numerical calculations; the dashed lines are approximations by linear dependencies. For large $n$, nonlinear effects begin to appear.

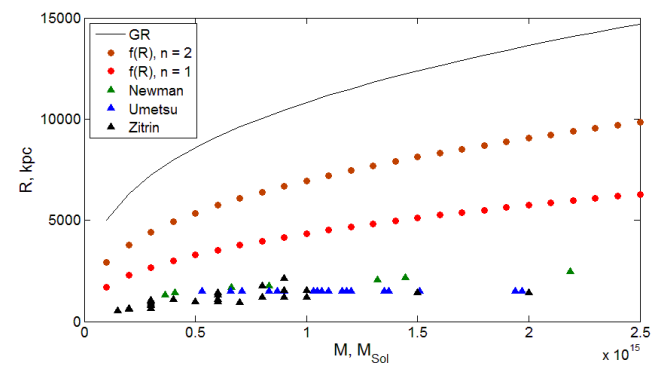

Figure 3. Comparison of the theoretical values of the turning radius with those obtained from observations of masses of clusters. The line denotes the values given by GR. The values for the Starobinsky models with $n=1$ and $n=2$ are denoted by round points, the values for $n=2$ are above. Real data values are indicated by triangles. 


\section{References}

[1] S.O. Alexeyev, B.N. Latosh, V.A. Echeistov, J. Exp. Theor. Phys. 125, 1083 (2017), [Zh. Eksp. Teor. Fiz.152,no.6,1271(2017)]

[2] C.M. Will, Living Rev. Rel. 17, 4 (2014), 1403.7377

[3] B.P. Abbott et al. (Virgo, LIGO Scientific), Phys. Rev. Lett. 116, 061102 (2016), 1602.03837

[4] B.P. Abbott et al. (Virgo, LIGO Scientific), Phys. Rev. Lett. 116, 221101 (2016), 1602.03841

[5] P.A.R. Ade et al. (Planck), Astron. Astrophys. 594, A13 (2016), 1502.01589

[6] Ya.B. Zel'dovich, A. Krasinski, Ya.B. Zeldovich, Sov. Phys. Usp. 11, 381 (1968), [Usp. Fiz. Nauk95,209(1968)]

[7] S. Weinberg, Rev. Mod. Phys. 61, 1 (1989)

[8] M.H. Goroff, A. Sagnotti, Nucl. Phys. B266, 709 (1986)

[9] C.P. Burgess, Living Rev. Rel. 7, 5 (2004), gr-qc/0311082

[10] A.D. Linde, Lect. Notes Phys. 738, 1 (2008), 0705.0164

[11] S. Capozziello, M. De Laurentis, Phys. Rept. 509, 167 (2011), 1108.6266

[12] T. Clifton, P.G. Ferreira, A. Padilla, C. Skordis, Phys. Rept. 513, 1 (2012), 1106.2476

[13] E. Berti et al., Class. Quant. Grav. 32, 243001 (2015), 1501.07274

[14] A. De Felice, S. Tsujikawa, Living Rev. Rel. 13, 3 (2010), 1002 . 4928

[15] V. Faraoni, Phys. Rev. D72, 124005 (2005), gr-qc/0511094

[16] A.A. Starobinsky, JETP Lett. 86, 157 (2007), 0706. 2041

[17] A.A. Starobinsky, Phys. Lett. B91, 99 (1980)

[18] C. Charmousis, Lect. Notes Phys. 892, 25 (2015), 1405. 1612

[19] J. Ntahompagaze, A. Abebe, M. Mbonye, Int. J. Geom. Meth. Mod. Phys. 14, 1750107 (2017), 1706.07722

[20] R. Nandra, A.N. Lasenby, M.P. Hobson, Mon. Not. Roy. Astron. Soc. 422, 2931 (2012), 1104.4447

[21] V. Faraoni, Phys. Dark Univ. 11, 11 (2016), 1508.00475

[22] J. Kormendy, D.B. Fisher, M.E. Cornell, R. Bender, Astrophys. J. Suppl. 182, 216 (2009), 0810.1681

[23] B. Binggeli, Astronomy and Astrophysics 107, 338 (1982)

[24] A.I. Zabludoff, M.J. Geller, Astron. J. 107, 1929 (1994)

[25] R.B. et al., IEEE Transactions on Electron Devices 39, 1031 (1983)

[26] M. Fich, S. Tremaine, Ann. Rev. Astron. Astrophys. 29, 409 (1991)

[27] A. Zitrin et al., Astrophys. J. 801, 44 (2015), 1411. 1414

[28] K. Umetsu et al., Astrophys. J. 795, 163 (2014), 1404. 1375

[29] A.B. Newman, T. Treu, R.S. Ellis, D.J. Sand, C. Nipoti, J. Richard, E. Jullo, Astrophys. J. 765, 24 (2013), 1209. 1391 